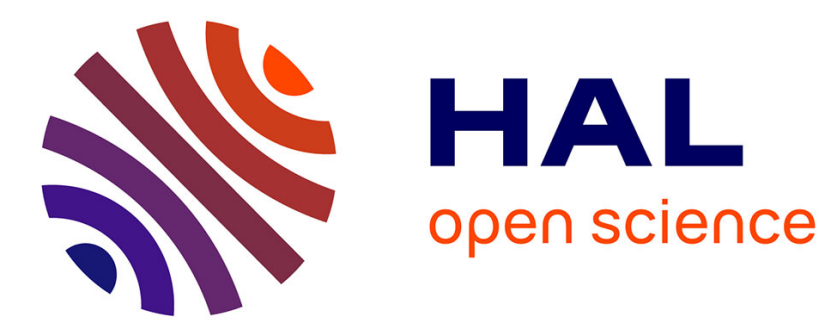

\title{
What Can the Concept of a Perfect Chemoelastic Solid Tell us about the Mechanical and Thermodynamic Behaviour of a Solid?
}

\author{
F. Larché
}

\section{- To cite this version:}

F. Larché. What Can the Concept of a Perfect Chemoelastic Solid Tell us about the Mechanical and Thermodynamic Behaviour of a Solid?. Journal de Physique IV Proceedings, 1996, 06 (C1), pp.C1-03-C1-09. 10.1051/jp4:1996101 . jpa-00254132

HAL Id: jpa-00254132

https://hal.science/jpa-00254132

Submitted on 1 Jan 1996

HAL is a multi-disciplinary open access archive for the deposit and dissemination of scientific research documents, whether they are published or not. The documents may come from teaching and research institutions in France or abroad, or from public or private research centers.
L'archive ouverte pluridisciplinaire HAL, est destinée au dépôt et à la diffusion de documents scientifiques de niveau recherche, publiés ou non, émanant des établissements d'enseignement et de recherche français ou étrangers, des laboratoires publics ou privés. 


\title{
What Can the Concept of a Perfect Chemoelastic Solid Tell us about the Mechanical and Thermodynamic Behaviour of a Solid?
}

\author{
F.C. Larché \\ Université Montpellier 2, case 26, 34095 Montpellier cedex 5, France
}

\begin{abstract}
The elastic energy stored in a solid is typically small compared to the energy involved in a phase change. As a consequence, it is relatively easy to generate elastic stresses by a modification of the composition and/or a phase transformation. Conversely, the influence of stresses on phase changes is only of importance in special situations. What kind of effects can we expect and what are the order of magnitude of the phenomena is the question which is addressed in this communication. Using a model of a perfect chemoelastic crystalline solid, developed in collaboration with J.W. Cahn many years ago, the various facets of the interactions between stresses and thermodynamics are reviewed. Besides a purely energetic action, the long range nature of the elastic forces and their anisotropy have unusual consequences for the material scientist. Thus because the global elastic energy in a self-stressed system undergoing a phase change is shape dependant, there is a coupling between elastic and surface effects, both contributing to the evolution of the shape of precipitates. The thin film geometry is very useful for the exploration of the new phenomena induced on equilibrium and kinetic evolution of multiphase solids by the presence of such stresses. Limitations of this model due to interactions with cracks or dislocations are briefly discussed.
\end{abstract}

\section{INTRODUCTION}

Solid-solid phase transformations have now been studied for more than hundred years [1]. The similarities of the phenomena observed in such materials and in liquids was striking. All the properties and the rules found with fluids were very quickly adopted for solids. The possible interactions with nonhydrostatic stresses were mentioned here and there (see [2-9] and references therein), but were usually forgotten in the next decade. The energetic considerations to follow show indeed that the order of magnitude of the effects can easily make them negligible. Moreover, many mechanisms can interact to relieve the stress and thus eliminate its influence on phase transformations. This can easily explain the history of this field. The much widespread use of single crystals, especially in the electronic industry, the availability of the necessary data and the increase of computer power gave a new impetus to the field. It has also been realised that stress is an essential ingredient in the understanding of such phenomena as diffusion induced grain boundary motion or diffusion induced liquid film migration $[10,11]$. Applied stresses have been shown to trigger phase transitions [12]. It is also now accepted that stresses are in part responsible for the microstructure development in materials and computer simulations have reproduced quite closely some of the observed microstructures [13].

In view of the much complex theory of phase transitions when non-hydrostatic stresses are taken into consideration, this presentation has been organised as followed. First, the order of magnitude of the energies involved will be assessed. From such an analysis, conditions under which stresses might be important are described. Then the model of an ideal chemoelastic solid is presented. It is used to show some of the unusual consequences of stresses on solid-solid phase transformation. We shall end on a discussion of the phenomena that could also interact to reduce the strain energy of the system and thus perturb the image given by the preceding description. 


\section{ENERGETIC CONSIDERATIONS}

It is instructive to compare the various types of free energy involved in solid-solid phase transformations. To do so, we have taken typical orders of magnitude and converted them in term of the thermal energy per atom $\mathrm{kT}$, where $\mathrm{k}$ is Boltzman constant and $\mathrm{T}$ the absolute temperature. The following values are found (see appendix for the details of the calculations):

- a mixing of a pure component in an alloy producing a change of molar fraction of $1 \%: 2.510^{-2} \mathrm{kT}$, - a phase change without change of composition at $10 \mathrm{~K}$ below the transition temperature: $210^{-2} \mathrm{kT}$, - the elastic energy of a uniaxially stressed solid, with a $0.5 \%$ strain: $210^{-3} \mathrm{kT}$,

- the interfacial free energy of a spherical particle of $1 \mu \mathrm{m}$ diameter: $210^{-3} \mathrm{kT}$.

Strain and interfacial energies are of the same order of magnitude and an order of magnitude smaller than the two others. Assuming coupling between these different kinds of energy - and it usually exists interesting consequences can be deduced from these simple observations.

The first is that, as a rule, elastic energy has a negligible influence on phase transformations. As an example, the change of phase composition from incoherent to coherent equilibrium is often small, albeit measurable [14]. The same conclusion is reached on the effects of externally applied stresses [15]. The analysis of the phase transformation can be made independently of elastic considerations, which is a great simplification. This hypothesis is similar to the use of an uncoupled heat equation in thermoelasticity [16]. It also explains why fluid thermodynamics was so successful in treating phase transformations in solids.

Conversely, there is enough energy available from composition and phase changes to give rise to very large stresses. Plastic deformations or cracks are common consequences on materials. Under the hypotheses of purely elastic deformations, the results of the phase transformation analysis can directly be used to calculate the stress field. The similarities with thermoelasticity are again obvious. However the stress level easily reaches the limit of elasticity. The hypothesis of a purely elastic behaviour breaks down and stress releasing mechanisms become operative. Dislocation multiplication is the most common in metals and is easily observed in diffusion fields (see for instance references in [17]). Because they constitute easy diffusion paths and increase the density of vacancies sources and sinks, they could modify the kinetics of the transformation. In materials with little plasticity, cracks are likely to appear. The resulting evolution of the geometry, the disruption of the diffusion paths in the solid and the multiplication of surface of discontinuity may have, in turn, a profound influence on the phase transformation [18]. These indirect effects may clearly be as important as more direct ones and deserve attention.

Both the elastic and the total interfacial energy of a precipitate are shape dependant. Their order of magnitude is similar. As a consequence one can expect a simultaneous influence on the microstructure of multiphase solids. More precise results have been obtained theoretically [19]. The effects observed experimentally are quite important [20].

The preceding analysis applies to the generic case. It can be pushed further by searching conditions for which elastic energy might become dominant. A very famous one is the neighbourhood of a critical point. Here the free energy as a function of composition is very flat and the boundaries of the phase diagram are strongly modified by the internal strain energy due to the different atomic sizes of the components of the solid solution. This is the well known lowering of the critical point due to coherency strains [21]. The elastic energy can also reach locally much higher levels than on a macroscopic scale. This is the case near defects like the tip of a crack or the core of a dislocation. Phase transformation can occur at these locations, the rest of the solid remaining untransformed [22]. Elastic and interfacial energies have different evolution with the size of precipitates, varying respectively like the volume and the area. At small size, interfacial energy dominates and there is a crossover to elastic control at sufficiently large size. Computations have clearly shown such bifurcations [23]. The size used in the above order of magnitude calculations correspond to this crossover region. Thus even at the qualitative level of a purely energetic analysis, there exist conditions under which elastic effects are important. To further assess the influence of stresses on solid-solid phase transformations, we now consider one of the simplest model of a multicomponent, multiphase crystal: the ideal chemoelastic solid.

\section{THE IDEAL CHEMOELASTIC SOLID}

An ideal chemoelastic solid has no stress releasing mechanisms other than composition or phase changes. We shall further assume here for simplification that the deformations are sufficiently small to use the small strain approximation and that the temperature is constant. As one tries to construct a theory of 
phase equilibrium, starting from a Helmoltz free energy depending only on strain and composition, it becomes quickly apparent that further ingredients are necessary [9]. The first is to realise that atoms in a crystalline solid cannot move at will. The number of lattice sites in a reference configuration is fixed. The final result of a change of composition is an exchange of atoms. This is the basis of the network concept $[24,25]$. The second is the necessity of a model for the interface between two phases, even when the energetic aspect of this interface (interfacial free energy and tension) is neglected. Among the various possible models, two extreme cases are of interest. The coherent interface imposes strong conditions between the crystals on each side. As a result, it can support any state of stress. The theoretical developments concerning phase equilibrium along such an interface were first published by Robin [8] and later by Larché and Cahn [9], but the key ingredients can already be found in Eshelby [26]. Some of these conditions are relaxed on an incoherent interface. In the "well greased" model [9], there is just contact between the two phases and only normal tractions remain.

There is no further requirement to develop the general conditions of equilibrium in a multicomponent stressed solid. They are obtained through standard variational calculus. They can be classified as mechanical when they are related to a displacement variation and to chemical when composition or phase change are involved. There are of two kinds: bulk and boundary conditions. For the following discussion, we consider that the two phases are substitutional binary solid solutions. The bulk chemical condition expresses the system equilibrium with respect to exchange of atoms. It imposes that the derivative of the Helmholtz free energy with respect to composition is constant. This derivative has been called diffusion potential. There is only one diffusion potential in a binary system. Within the present hypothesis, its expression is [27]

$$
\mathrm{M}_{12}\left(\sigma_{\mathrm{ij}}, \mathrm{c}\right)=\frac{\partial \mathrm{G}}{\partial \mathrm{c}}-\mathrm{V}_{0}^{\prime} \eta \sigma_{\mathrm{kk}}+\frac{\mathrm{V}_{0}^{\prime}}{2}\left[\frac{\mathrm{d}}{\mathrm{dc}}\left(\frac{\mathrm{v}}{\mathrm{E}}\right)\left(\sigma_{\mathrm{kk}}\right)^{2}-\frac{\mathrm{d}}{\mathrm{dc}}\left(\frac{1+v}{\mathrm{E}}\right) \sigma_{\mathrm{ij}} \sigma_{\mathrm{ij}}\right]
$$

for an isotropic solid and

$$
M_{12}\left(\sigma_{i j}, c\right)=\frac{\partial G}{\partial c}-V_{0}^{\prime} \eta_{i j} \sigma_{i j}+\frac{1}{2} V_{0} \frac{d S_{i j k l}}{d c} \sigma_{i j} \sigma_{k l}
$$

for an anisotropic solid. 1 and 2 are the constituents of the solid solution, $\sigma_{\mathrm{ij}}$ are the components of the stress tensor, $\mathrm{c}$ is the mole fraction of component $1, \mathrm{G}$ is the molar Gibbs free energy of the solution under negligible pressure, $V_{0}^{\prime}$ is the molar volume in the state chosen as reference for the strain, $\eta$ is the chemical expansion coefficient, $v$ is Poisson's ratio, $\mathrm{E}$ is Young's modulus, $\eta_{\mathrm{ij}}$ are the components of the chemical expansion tensor and $\mathrm{S}_{\mathrm{ijkl}}$ are the components of the compliance tensor.

Expression (1) and (2) contain three different types of terms. The first, dG/dc, is a purely chemical term. It is easily computed from standard Gibbs free energy measurements by making a small correction for non-zero pressure[27]. It is the only contribution in the absence of stress effects.

The second, $V_{0}^{\prime} \eta \sigma_{\mathrm{kk}}$ or $V_{0}^{\prime} \eta_{\mathrm{ij}} \sigma_{\mathrm{ij}}$, results from a coupling between the deformation produced by a local change of composition and the stress. It is linear in stress. In solids with isotropic or cubic symmetry, the chemical expansion is isotropic. Coupling involves only the trace of the stress tensor. This term will tend to increase the concentration of the biggest atoms in the regions of tension (i.e. where the trace of the stress tensor is positive) and increase the concentration of the smallest atoms in the regions of compression (i.e. where the trace of the stress tensor is negative). It is this term which is used in theories where atoms are considered as centres of dilatation [28-30]. In crystals with other symmetries than cubic, there are interactions with other components of the stress tensor than its trace. It is then conceivable to have an increase of the concentration of the largest atoms in regions of compression. The last terms of equation (1) and (2) indicate an other coupling between composition and stress. It arises from the variations of the elastic coefficients with composition. It is non linear in stress.

The chemical expansion coefficient can easily be very large compared to the strains involved in small strain theory. Values of the order of a few percent are common. Taking also a few percent as an order of magnitude for the change in elastic coefficient with composition and a stress of E/100, one can conclude that the quadratic terms are usually negligible with respect to the linear terms.

We just discussed the equation pertaining to bulk chemical equilibrium. It does not involve phase change. It is internal equilibrium within each phase on a local basis. The second chemical equation of equilibrium appears only along the interface. It reads

$$
\omega^{\alpha}-\omega^{\beta}-v_{0}^{, \alpha} n_{i}^{\alpha} \sigma_{i j}^{\alpha}\left(u_{j, k}^{\alpha}-u_{j, k}^{\beta}-u_{j, k}^{T(\alpha \rightarrow \beta)}\right) n_{k}^{\alpha}=0
$$


where $\alpha$ and $\beta$ are the phases in equilibrium, and quantities with superscript $\alpha$ or $\beta$ pertain respectively to the $\alpha$ or $\beta$ phase. $\omega$ is related to the grand canonical free energy per mole, $\varepsilon_{\mathrm{ij}}$ are the components of the strain tensor measured with respect to the stress free material, $u_{i, j}$ are the components of the

displacement gradient, $n_{i}^{\alpha}$ are the components of the normal to the interface directed from $\alpha$ to $\beta$, and the tensor components with superscript $T$ correspond to the transformation from stress free $\alpha$ to stress free $\beta$. This equation relates the jump in the normal derivative of the displacement gradient, when the same reference state is chosen for both phases, to the jump in $\omega$ function. Because of the coherence of the interface, this equation can also be written [31]

$$
\omega^{\alpha}-\omega^{\beta}-V_{0}^{, \alpha}\left[\sigma_{i j}^{\alpha} \varepsilon_{i j}^{\alpha}-\sigma_{i j}^{\beta}\left(\varepsilon_{i j}^{\beta}+\varepsilon_{i j}^{T(\alpha \rightarrow \beta)}\right)\right]=0
$$

In the case of a displacive transformation, one can consider the material as a one constituent system. $\omega$ is then equal to the Helmoltz free energy. In a binary substitutional solutions and elastically isotropic materials, its expression is

$$
\omega=\mathrm{f}-\mathrm{M}_{12} \mathrm{c}=\mu_{2}+\mathrm{V}_{0}^{\prime}\left[-\frac{v}{2 \mathrm{E}}\left(\sigma_{\mathrm{kk}}\right)^{2}+\frac{1+v}{2 \mathrm{E}} \sigma_{\mathrm{ij}} \sigma_{\mathrm{ij}}+\mathrm{c} \eta \sigma_{\mathrm{kk}}\right]
$$

where $\mathrm{f}$ is the molar Helmoltz free energy and $\mu_{2}$ is the chemical potential of component 2 at vanishing pressure (the term due to the variation of elastic coefficients with composition has been neglected).

Examination of equations (4) and (5) indicates that all the components of the stress tensor appear for phase equilibrium at the interface. This is in contrast to the diffusion potential of isotropic materials, where shear plays no role. We also note that elastic coefficients appear in this equation: the so called modulus effect is included in this equation. Even in the absence of interfacial energy, equation (4) governs the evolution of the interface. Equation (4) takes a particularly simple form in one component solids

$$
\left(\mu^{\beta}-\mu^{\alpha}\right) / V_{0}^{, \alpha}=\frac{1}{2} \sigma_{i j}^{\beta} \varepsilon_{i j}^{\beta}-\frac{1}{2} \sigma_{i j}^{\alpha} \varepsilon_{i j}^{\alpha}+\sigma_{i j}^{\alpha} \varepsilon_{i j}^{\Upsilon(\alpha \rightarrow \beta)}
$$

The jump in the chemical potential (per unit volume in the reference state) is equal to the jump in elastic energy density plus an elastic work term due to the jump in displacement gradient.

The full equilibrium problem involves two other equations, the elastic equilibrium equation

$$
\sigma_{\mathrm{ij}, \mathrm{j}}=0
$$

and the elastic boundary condition

$$
\sigma_{i j}^{\alpha} n_{j}^{\alpha}=-\sigma_{i j}^{\beta} n_{j}^{\beta}
$$

which indicates no jump in traction across a coherent interface.

It may be important to note that the previous equations are necessary conditions for an extremum in the appropriate free energy, but they do not guaranty a minimum. The fact that under no external load a uniform one phase solid is always stress free may easily make the solution with two phases and a coherent interface a metastable or an unstable one.

\section{SOME RESULTS ON IDEAL CHEMOELASTIC SOLIDS}

A constant diffusion potential, elastic equilibrium equations ( 7 and 8 ) and the phase equilibrium equation (4) are the necessary equations for the equilibrium of a binary ideal chemoelastic solid with two phases separated by a coherent interface. The characteristics of such an equilibrium are obviously different from the characteristics of an equilibrium between fluids. In the later, the composition of each phase gives a complete description. The proportions of the phases is just a result of the conservation of mass. In the former, the stress field, composition field and shape of the interface are necessary for a complete description. The search for the solutions of such problems combines several difficulties. There is coupling between elasticity and composition, it is a free boundary problem and the equations are nonlinear. Elastic anisotropy is an other complication. In view of such a complexity, simplifications are important.

The fact that stress effects are often small can be put in good use in the search for solutions of the aforementioned equations. They can be linearised around the composition of an equilibrium state with no stress (negligible pressure usually). This technique leads to the concept of elastic coefficients at constant diffusion potential also called open system elastic constants [26, 32]. Under a constant diffusion 
potential, a one-phase solid behaves, in the long time limit, as if it had new elastic properties. An applied load produces an elastic strain, in the short time limit, and a further strain is added due to the slower change of composition. Overall, the solid is more compliant. This linearisation uncouples the elastic and the composition field equations, making the solutions of many problems a matter of literature search in the solutions of elastic equations. This concept is more general than the above technique would imply. It can be developed independently as a specific partial derivative of the free energy function. Non-linear stress-strain curves are predicted [25] which could lead to instabilities and phase changes in regions of high stresses.

To find the equilibrium shapes of isolated precipitates requires a bigger effort. Progress has been made through the simplifications brought by a linearisation of the chemical part of the jump condition (4) and the addition of a surface energy term (a necessary ingredient to remain realistic). Solutions have been obtained in two dimensions for solids with cubic symmetry by Thompson et al. [33]. These numerical calculations show the evolution of the equilibrium shape with the size of the particle, indicating clearly the changing competition between elastic and interfacial energy.

An other complex area where work has been pursued along these lines is the microstructure evolution of alloys with coherent precipitates. Johnson and Voorhees [34-39] have made extensive investigations on Oswald ripening. In such studies, the boundary conditions of the kinetic equation is equation (4) suitably modified for interfacial effect. It becomes a Gibbs-Thomson equation with elastic terms. Most of the work done involves numerical calculations in two dimensions. The stresses affect both the morphology of individual particles and their spatial distribution [38]. Spatial correlations can appear from coarsening of an alloy with randomly distributed precipitates.

There are extreme simplifications with the thin plate geometry when the spatial distribution and loading conditions are such that stress and strain are uniform within each phase. Many of the theorems valid for liquid-liquid equilibria, and which are not valid for solids, can be extended. The notion of phase diagrams becomes meaningful and their properties have been explored [40-42]. Among the results, a new type of phase behaviour has been predicted near congruent points, extending earlier work on this subject [43-45]. At temperatures between the congruent transformation point and the William's point, there is disappearance of the two-phase region. Within this range, a change of temperature or composition of the alloy produces a direct transformation from one pure phase to the other. Order of magnitude calculations [41] predict such behaviour to be experimentally observable. With thin plates, calculations are amazingly simple and many complexities can be added [46]. It is extremely useful to assess possible effects and order of magnitude. However such a geometry may not always be valid as a simplified description of the phase behaviour of coherent solids since it may not be stable [47-49].

Equilibrium conditions can also be used as a basis for a kinetic theory with gradient flow [27]. The very different time scale for elastic and chemical equilibrium justifies the assumption of elastic equilibrium at all times. The resulting equations, for self-stressed solids, present interesting peculiarities $[50,51]$ due to the long range nature of elastic forces. The flux at a point becomes a functional of the composition field, substantially changing the composition field and its evolution with time.

\section{DISCUSSION AND CONCLUSION}

Predicting the evolution of the microstructure of an alloy as a result of processing conditions is one of the goal of the material scientist. The theory presented in the preceding sections has, in principle, all the ingredients to do so. However its application forces to track the interfaces as they evolve. This is often a difficult computational task. A continuous counterpart which circumvents these problems has also been proposed. Originating from van der Waals [52], introduced by Cahn and Hilliard in materials science [53], it has been much applied in recent years. The interface is replaced by a gradient energy term in the free energy density. In the limit of infinitely large gradients, the two theories converge. The location of the interfaces is obtained as a level surface in composition and a phase change does not produce any discontinuity. Such a model automatically includes surface energy effects and it has often been used as such without the inclusion of strain energy. The formation and the evolution of the microstructure can be followed from numerical calculations. There is however a price to pay, as the number of grid points has to be sufficient in the region of high gradient. As a result such calculations are usually made in two dimensions. References [53-55] are examples on coarsening. It also implies the same crystal structure in both phases, a condition necessarily applied when isotropic elasticity is used, but more restrictive for anisotropic systems.

In this brief review, only small strain was considered. This is a very good approximation in the description of the elastic behaviour of most materials, but it can be more questionable when deformations due to composition changes or phase transformations are involved. A model involving the full strain tensor has been developed along the same lines as the small strain model presented here $[9,25]$. 
Non-linear effects have been predicted which can be of importance in regions of high stress concentration like close to a dislocation core.

A limitation of the ideal elastochemical solid is the presence of stress releasing mechanisms, particularly at high stresses. In metals, dislocation multiplication is probably the most common of these mechanisms. Besides reducing the stress to much lower levels on the average, this increase in dislocation density may have in turn other interactions with the process of phase transformation. Near the core, the level of stress remains very high. Even if the volume concerned is small, this may affect the course of the transformation by providing easy nucleation sites or even totally eliminating the nucleation barrier [5657]. An important density of dislocation may also influence the diffusion process and thereby the phase evolution. These indirect effects have not been much studied and work is needed in this area before an evaluation of their importance can be made. In brittle solids, high stresses are likely to produce cracks, thereby destroying the integrity of the material and introducing new surfaces of discontinuity. If such mechanisms are operative, it is obvious that they will have a profound influence on the course and the results of any phase transformation. To our best knowledge, almost nothing has been done from a theoretical point of view in this domain.

The theory has been constructed for single crystals but materials are more often used as polycrystals. The presence of grain boundaries strongly increases the complexity of model development. Here again a comprehensive theory has yet to be formulated.

The mechanical behaviour of materials which undergo phase transformation has just been briefly evoked when the concept of open systems elastic coefficients was presented. This is just one little side of the story, as under an externally applied load, a solid can also respond by phase change and microstructure evolution. The equations of equilibrium are enough to describe the micromechanical side, but we are far from a macroscopic description of the stress-strain behaviour, even in the absence of compositional effects. This other side of phase transformations in solids is rarely considered in material science and should deserve more attention.

Granted these various shortcomings, several conclusions can be derived from the model. 1. Overall effects are small. Evaluation can easily be made with the simple thin plate geometry.

2. Stresses can be heterogeneous and large locally. The total energy remains small, but the local diffusion potential is substantially modified as well as the $\omega$ function. The effect on phase change will also be substantial. This is probably what is seen in partially stabilised zirconia, when the quadratic to monoclinic transformation occurs near a propagating fracture tip [58-59].

3. Effects are important on microstructure development and compete with capillary effects.

4. The long range nature of elastic forces can be at the origin of unusual phenomena, as predicted on unsupported thin films [60].

\section{APPENDIX}

The order of magnitude of the free energy changes have been obtained using the following data and hypotheses. Temperature is $\approx 1000 \mathrm{~K}$

Change of composition: ideal solid solution, average atom fraction 0.11 in component 2 , original state: solution of composition 0.10 and pure 2 .

Phase change : $\Delta \mathrm{G}=\Delta \mathrm{H}\left(\mathrm{T}-\mathrm{T}_{\mathrm{e}}\right) / \mathrm{T}_{\mathrm{e}}$, where $\mathrm{T}_{\mathrm{e}}$ is the equilibrium temperature and $\mathrm{T}$ is the temperature of the phase change. Equilibrium temperature $1000 \mathrm{~K}$, enthalpy of the transformation $\Delta \mathrm{H}=16 \mathrm{~kJ} / \mathrm{mol}$.

Elastic energy: Isotropic solid under uniaxial strain $\mathrm{V}_{\mathrm{m}} E \varepsilon^{2} / 2$, where $\mathrm{V}_{\mathrm{m}}$ is the molar volume.

Interfacial energy: for a spherical particle, $3 \gamma \mathrm{V}_{\mathrm{m}} / \mathrm{r}$. Radius $\mathrm{r}=1 \mu \mathrm{m}$, interfacial free energy $\gamma=400 \mathrm{~mJ} / \mathrm{m}^{2}$.

\section{References}

[1] Roberts-Austen W. and Stansfield, A., "La constitution de alliages métalliques", Congrès International de Physique, Paris 1900, C.-E. Guillaume and L. Poincaré Eds, (Gauthier-Villars, Paris) pp. 363-401 and references therein.

[2] Bridgman P.W., Phys. Rev. 7 (1916) 215-223.

[3] Nabarro F.R.N., Proc. Roy. Soc. A175 (1940) 519-538.

[4] Patel J.R. and Cohen M., Acta Metall. 1 (1953) 531-538.

[5] Kamb W.B., J. Geophys. Res. 66 (1961) 259-271

[6] Cahn J.W., Acta Metall. 10 (1962) 907-913.

[7] Li J.C.M., Oriani, R.A. and Darken L.S., Zeit. Phys. Chem. Neue Folge 49 (1966) 271-290.

[8] Robin P.-Y., Amer. Miner. 59 (1974) 1286-1298.

[9] Larché F.C. and Cahn, J.W., Acta Metall. 26 (1978) 1579-1589. 
[10] Hillert M. and Purdy G.R., Acta Metall. 26 (1978) 333-340.

[11] Kim J.K. and Yoon D.Y., Acta Metall. Mater. 42 (1994) 913-919.

[12] Evans A.G. and Heuer A.H., J. Amer. Ceram Soc. 63 (1980) 241-248.

[13] Wang Y., Chen L.-Q. and Khatchaturyan A., "Computer simulation of microstructure evolution in coherents solids", Solid $\rightarrow$ solid phase transformations, Farmington, Pa. 1994, W.C. Johnson, J.M. Howe, D.E. Laughlin and W.A. Soffa Eds. (TMS, Warrendale, Pa. 1994) pp. 245-265.

[14] Grujicic M. and Olson G.B., CALPHAD 12 (1988) 405-414.

[15] Larché F.C. and Cahn J.W., J. Appl. Phys. 62 (1987) 1232-1239.

[16] Boley B.A. and Weiner J.H., Theory of thermal stresses (John Wiley ans Sons, Inc, New-York, 1960).

[17] Li J. C.-M., Metall. Trans. 9A (1978) 1353-1380.

[18] Bertrand G., Solid State Phenomena 3-4 (1988) 257-266.

[19] Johnson W.C. and Cahn J.W., Acta Metall. 32 (1984) 1925-1933.

[20] Miyazaki T., Inamura H. and Kozakai T., Mat. Sc. Eng. 54 (1982) 9-15.

[21] Cahn J.W., Acta Metall. 10 (1962) 907-913.

[22] Xiao S.Q., Wilbrandt P.-J. and Haasen, P., Scripta Metall. 23 (1989) 295-300.

[23] Johnson W.C. and Cahn J.W., Acta Metall., 32 (1984) 1925-1933.

[24] Larché F.C. and Cahn J.W., Acta Metall. 21 (1973) 1051-1063.

[25] Larché F.C. and Cahn J.W., Acta Metall. 26 (1978) 53-60.

[26] Eshelby, J.D., "Energy relations and the energy-momentum tensor in continuum mechanics", in Inelastic Behavior of Solids, Kanninen, M.F., Adler, W.F., Rosenfeld, A.R., Jaffee, R.I., Edts, (McGrawHill, 1970) pp. 77-115.

[27] Larché F.C. and Cahn J.W., Acta Metall. 33 (1985) 331-357.

[28] Gorsky W.S., Phys. Zeitschr. Sow., 8 (1935) 562.

[29] Cottrell A.H., "Effect of solute atoms on the behaviour of dislocations", Report of a conference on strength of solids, Bristol 1947 (The Physical Society, London, 1948) pp. 30-38.

[30] Eshelby J.D., J. Applied Phys. 25 (1954) 255-261.

[31] Gurtin M.E. and Voorhees P.W., Proc. R. Soc. Lond. A 440 (1993) 323-343.

[32] Larché F.C. and Cahn J.W., Acta Metall. 21 (1973) 1051-1063.

[33] Thompson M.E., Su C.S. and Voorhees P.W., Acta Metall. Mater. 42 (1994) 2107-2122.

[34] Johnson W.C., Voorhees P.W. and Zupon D.E., Metall. Trans. 20A (1989) 1175.

[35] Voorhees P.W. and Johnson W.C., Phys. Rev. Lett. 61 (1988) 2225-2228.

[36] Johnson W.C., Abinandanan T.A. and Voorhees P.W., Acta Metall. 38 (1990) 1349-1367.

[37] Laria V.J., Johnson W.C. and Voorhees P.W., Scripta Metall., 23 (1989) 1749-1754.

[38] Johnson W.C. and Voorhees P.W., Solid State Phenomena 22-24 (1992) 87-103.

[39] Voorhees P.W., Ann. Rev. Mat. Sci. 22 (1992) 197-215.

[40] Johnson W.C. and Müller W.H., Acta Metall. Mater. 39 (1991) 89-103.

[41] Pfeifer M.J. and Voorhees P.W., Met. Trans. 22A (1991) 1921-1935.

[42] Liu Z.-K. and Ågren J., Acta Metall. Mater. 38 (1990) 561.

[43] Williams R.O., Met. Trans. 11A (1980) 247-253.

[44] Williams R.O., CALPHAD 8 (1984) 1-14.

[45] Cahn J.W. and Larché F.C., Acta Metall., 32 (1984) 1915-1923.

[46] Lee J.K. and Tao W., to be published.

[47] Srolovitz D.J., Acta Metall., 37 (1989) 621-625.

[48] Grinfeld M.A., Soviet Phys. Dokl., 31 (1986) 831-834.

[49] Junqua N. and Grilhé J., Phil. Mag. Letters 69 (1994) 61-70.

[50] Larché F.C., "Diffusion sous gradient de contraintes - éléments de théorie et leurs applications", 19ème colloque de métallurgie, Saclay 1976, (CEA, Saclay, 1977) pp. 989-1002.

[51] Larché F.C., Solid State Phenomena 3-4 (1988) 205-214.

[52] van der Waals J.D., translation by Rowlinson J.S., J. Stat. Phys. 20 (1979) 197-244.

[53] Cahn J.W. and Hilliard J.E., J. Chem. Phys. 28 (1958) 258-267.

[54] Nishimori H. and Onuki A., Phys. Rev. B 42 (1990) 980-983.

[55] Wang Y., Chen L.Q. and Khatchaturyan A.G. Scr. Metall. Mater. 25 (1991) 1969.

[56] Larché F.C., in Dislocations in solids, Nabarro F.R.N., Ed. (North-Holland, Amsterdam 1979) vol.4, pp. 134-153.

[57] Larché F.C., "Thermodynamics of stressed solids, precipitation on dislocations", Dislocations 93 , Aussois 1993, J. Rabier, A. George, Y. Bréchet and L. Kubin Eds (SCITEC Publications 1994) pp. 173182.

[58] Evans G., J. Amer. Ceram. Soc. 73 (1990) 187-206.

[59] Lee R.-R. and Heuer A.H., J. Amer. Ceram. Soc. 71 (1988) 694-700.

[60] Larché F.C. and Cahn J.W., Acta Metall. Mater. 40 (1992) 947-955. 